\title{
Cue-dependent circuits for illusory contours in humans
}

Jacques Anken ${ }^{1}$, Jean-François Knebel ${ }^{2,3}$, Sonia Crottaz-Herbette ${ }^{1}$, Pawel J. Matusz ${ }^{1,4}$, Jérémie Lefebvre ${ }^{2,5}$, Micah M. Murray ${ }^{1-3,6,7, *}$

The Laboratory for Investigative Neurophysiology (The LINE), ${ }^{1}$ Department of Clinical Neurosciences and ${ }^{2}$ Department of Radiology, University Hospital Center and University of Lausanne, Lausanne, Switzerland

${ }^{3}$ Electroencephalography Brain Mapping Core, Center for Biomedical Imaging (CIBM) of Lausanne and Geneva, Switzerland

${ }^{4}$ Attention, Brain and Cognition Group, Department of Experimental Psychology, Oxford, UK

${ }^{5}$ Toronto Western Research Institute, Toronto, Canada

${ }^{6}$ Department of Ophthalmology, University of Lausanne, Jules-Gonin Eye Hospital, Lausanne, Switzerland

${ }^{7}$ Department of Hearing and Speech Sciences, Vanderbilt University, Nashville, TN, USA

\section{Address correspondence to:}

Prof. Micah Murray

CHUV-UNIL

Radiologie, BH08.078

Rue du Bugnon 46

1011 Lausanne

Micah.murray@chuv.ch 


\section{Abstract}

Objects' borders are readily perceived despite absent contrast gradients, e.g. due to poor lighting or occlusion. In humans, a visual evoked potential (VEP) correlate of illusory contour (IC) sensitivity, the "IC effect", has been identified with an onset at $\sim 90 \mathrm{~ms}$ and generators within bilateral lateral occipital cortices (LOC). The IC effect is observed across a wide range of stimulus parameters, though until now it always involved high-contrast achromatic stimuli. Whether IC perception and its brain mechanisms differ as a function of the type of stimulus cue remains unknown. Resolving such will provide insights on whether there is a unique or multiple solutions to how the brain binds together spatially fractionated information into a cohesive perception. Here, participants discriminated IC from no-contour (NC) control stimuli that were either comprised of low-contrast achromatic stimuli or instead isoluminant chromatic contrast stimuli (presumably biasing processing to the magnocellular and parvocellular pathways, respectively) on separate blocks of trials. Behavioural analyses revealed that ICs were readily perceived independently of the stimulus cue - i.e. when defined by either chromatic or luminance contrast. VEPs were analysed within an electrical neuroimaging framework and revealed a generally similar timing of IC effects across both stimulus contrasts (i.e. at $\sim 90 \mathrm{~ms}$ ). Additionally, an overall phase shift of the VEP on the order of $\sim 30 \mathrm{~ms}$ was consistently observed in response to chromatic vs. luminance contrast independently of the presence/absence of ICs. Critically, topographic differences in the IC effect were observed over the 110-160ms period; different configurations of intracranial sources contributed to IC sensitivity as a function of stimulus contrast. Distributed source estimations localized these differences to LOC as well as V1/V2. The present data expand current models by demonstrating the existence of multiple, cue-dependent circuits in the brain for generating perceptions of illusory contours.

Key words: illusory contour, Kanizsa, event-related potential (ERP), visual evoked potential (VEP), object recognition, magnocellular; parvocellular 


\section{Introduction}

The visual system can create perceptions of boundaries despite the visual input to the retina being discontinuous or incomplete; resulting from poor lighting, occlusion, or myriad other everyday situations. These perceptions, including ICs, have been the subject of extensive theoretical debate and experimental research across species (Murray and Herrmann, 2013). A commonly-used stimulus was popularized by Kanizsa (1976) and includes an array of circular sectors (pacmen), whose mouths are oriented so to induce ICs or, alternatively, rotated as to prevent such perceptions (hereafter nocontour; NC) (Figure 1).

Several competing models have been proposed regarding how the brain produces ICS (Murray and Herrmann, 2013). Some propose that low-level areas V1/V2 mediate IC sensitivity in a feed-forward manner (Grosof et al., 1993; Nieder and Wagner, 1999; Redies et al., 1986; von der Heydt et al., 1984). Others propose that lateral occipital cortices (LOC) within the ventral visual pathway (Ungerleider and Mishkin, 1982) mediate it, and that any effects in V1/V2 reflect feedback modulations subsequent to IC sensitivity itself (Lee and Nguyen, 2001; Mendola et al., 1999; Murray et al., 2006, 2004, 2002; Sáry et al., 2008, 2007). Still others propose that the LOC detects salient regions defined by the pacmen inducers, but that IC sensitivity is itself performed within V1/V2 albeit under the control of feedback modulations (from the LOC and elsewhere) (Hochstein and Ahissar, 2002; Stanley and Rubin, 2003; Yoshino et al., 2006).

A VEP correlate of IC sensitivity has been identified - the IC effect - that onsets at $~ 90 \mathrm{~ms}$ post-stimulus (i.e. during the P1/N1 components of the VEP) and is localised to the bilateral LOC (Murray et al., 2002). This IC effect has been observed across various manipulations of low-level stimulus features inducing ICs, including contrast polarity, eccentricity, types of inducers, and modal/amodal completion (Murray and Herrmann, 2013). However, a major shortcoming of all prior neurophysiologic studies of IC sensitivity in animals and humans is that the employed stimuli were invariably high in contrast (black and white, in fact). The extent to which the spatio-temporal brain 
dynamics of the IC sensitivity are cue-dependent and impacted by stimulus features, such as luminance and chromaticity, remains unknown. Resolving the role of these stimulus features in IC sensitivity would provide much-needed insights into potentially differing contributions of magnocellular versus parvocellular subdivisions of the visual system to IC sensitivity (Ejima and Takahashi, 1988; Gregory, 1977; Li and Guo, 1995; Soriano et al., 1996) as well as potentially reconcile the discrepant findings and the resultant models of IC sensitivity.

It has been suggested that mechanisms based on luminance and those based on chromaticity might both contribute to IC sensitivity, with the two mechanisms operating concurrently (Takahashi et al., 1992). At present, direct neurophysiologic support for this proposal is largely lacking. Indirect supporting evidence for has been provided by the results demonstrating that ICs can be induced with both static and moving inducers (e.g., Seghier et al., 2000) as well as with inducers that oscillate (Masuda et al., 2015). Such data would suggest that both dorsal and ventral visual pathways (which are thought to receive a preponderance of magnocellular and parvocellular inputs, respectively) likely contribute to IC sensitivity processes. In line with this suggestion, parietal sources have been identified as contributing to the IC effect (cf. Figure 6a in Murray et al., 2002; reviewed in Murray and Herrmann, 2013). However, parietal structures do not appear to forcibly be requisite for IC perception. Studies of brain-lesioned patients have shown that IC perception critically depends on the integrity of the LOC, but persists despite damage to parietal cortices (cf. Figure 5 in Vuilleumier et al., 2001). Similarly, studies of patients with schizophrenia would indicate that the IC effect triggered by high-contrast achromatic stimuli is indistinguishable from that recorded from healthy controls, despite severely impaired P1 component responses in the former group (Foxe et al., 2005; Knebel et al., 2011). More generally, evidence is accumulating to support the idea of impaired magnocellular system function in schizophrenia (Butler et al., 2007; Javitt, 2009). One implication for IC sensitivity is that this process might operate largely independently of the magnocellular and/or dorsal pathway, relying instead on the integrity of the parvocellular system and the ventral stream structures. However, and because extant studies have used high-contrast achromatic stimuli, it is not clear if IC 
processes operate in a cue-invariant manner. Data from recordings within lower-level visual cortices (V1/V2) in animals would suggest that orientation and contour sensitivity may operate in a largely cue-invariant manner (e.g. (Song and Baker, 2007; Gharat and Baker, 2012)).

In light of such, we reasoned that cue-invariant IC effects would be consistent with IC processing being mediated in a (largely) feed-forward manner by regions such as V1/V2. By contrast, cue-dependent IC effects would instead support LOC-centred models of IC processing. The current study thus determined whether the IC effect is limited to the specific type of stimulus contrast used to elicit it; the evidence for such limitation would undermine the emerging consensus on the (uniform) brain underpinnings of IC sensitivity. By analysing VEPs within an electrical neuroimaging framework, we differentiated effects arising due to changes in the brain response timing, strength, and topography (Michel and Murray, 2012; Murray et al., 2008). If differences were found merely in the strength of responses of a statistically indistinguishable network across the two IC contrasts, this would suggest that a single, uniform brain network/mechanism mediates IC sensitivity. If, instead, early differences in the topography and underlying sources were found between the two types of contrast, such a result - depending on how strong / early were the differences observed - could suggest a 1) certain flexibility within the already identified network or, alternatively, 2) separate and distinct brain circuits activated by different types of stimulus contrast. The latter would necessitate revision of the emerging consensus about the brain mechanisms giving rise to IC sensitivity.

\section{Material and Methods}

\subsection{Participants}

Analyses presented in this study are based on data from 12 participants (4 male, all righthanded; aged $23-33$ years, mean 25.8 years). All were post-graduate university students at the time of testing. No subject had history of or current neurological or psychiatric illness. All participants had normal or corrected-to normal vision and no problems with colour vision or colour-blindness were reported. The integrity of colour vision was based on participants' self-reports according to their 
prior experiences with the Ishihara colour test (Ishihara, 1972), as routinely performed in Swiss primary schools as well as in military recruiting centres. Data from an additional 8 subjects were excluded due to either excessive muscle and/or alpha frequency EEG artefacts $(\mathrm{N}=7)$ or technical issues with behavioural response recording during data acquisition $(\mathrm{N}=1)$.

\subsection{Stimuli and task}

Stimuli were comprised of a set of 4 circular Kanizsa-type (Kanizsa, 1976) 'pacmen' inducers that were arranged to either form an illusory contour or not (IC and NC conditions, respectively) (Figure 1). Each inducer subtended $1.26^{\circ}$ in diameter of visual angle at a distance of $150 \mathrm{~cm}$. On a given trial, the four pacmen were either positioned along the horizontal and vertical axes to form a circular IC (and its NC equivalent; $1.4^{\circ}$ centre-to-centre eccentricity) or, alternatively, were positioned along the $45^{\circ}$ diagonals to form a square IC (and its NC equivalent; $2.0^{\circ}$ centre-to-centre eccentricity). These variations in how ICs were created were included to prevent participants from selectively attending to particular regions of space as a strategy to successfully complete the task. The employed forms have been used in prior IC studies by our group and are known to result in similar IC sensitivity (Knebel and Murray, 2012; Knebel et al., 2011; Murray and Herrmann, 2013).

The IC stimulus features that were the main focus of our study were the chromatic versus low-luminance contrast of the pacmen vs. the background display (hereafter referred to as $\mathrm{C}$ and $\mathrm{L}$, respectively). C- and L-ICs were separately adjusted, as to generate stimuli biasing the processing predominantly towards the parvocellular and magnocellular visual pathways, respectively. For the measure of luminance, the following definition was used: $Y=0.2126 R+0.71524 G+0.0722 B$. (i.e. the luminance $Y$ is composed in $21.26 \%$ of Red, in $71.524 \%$ of Green and in $7.22 \%$ of Blue) (see Knebel et al., 2008). For the chromatic contrast condition, there were either green 'pacmen' against a pink background or the vice versa. The RGB values were for green: $R: 74, G: 146, B: 109(Y=128)$ and for pink: R:236, G:92, B:166 ( $Y=128)$. The Weber contrast (with the measurement on the screen) for the pink stimuli on a green background was $28 \%$, while for green stimuli on a pink background this value was $-22 \%$. The Weber contrast for the achromatic condition was $-0.11 \%$. The measurements were 
performed with a digital light meter TES-1332A. The luminance of the 'pacmen' and the background on the screen was equivalent and controlled with photographic techniques using the diaphragm aperture (Minolta Auto Meter IV F). The aperture was identical for the green, pink and the grey background values. The photographic aperture $f / N$ was on average $f / 5.6_{2}$ on the screen for each of the 3 colours. This value equals the ratio of the lens's focal length to the diameter of the entrance pupil. It is a dimensionless value indicative of lens speed. If the aperture is the same for two colours, the luminance of the colours is equivalent. This was the case here for the green and pink colours. For the low-luminance contrast condition, the RGB values of the inducers were R:125,G:125,B:125 $(\mathrm{Y}=125)$ and those of the background were $\mathrm{R}: 128, \mathrm{G}: 128, \mathrm{~B}: 128(\mathrm{Y}=128)$ (i.e. the 'pacmen' were slightly darker than the background). Stimuli were displayed on an LCD computer monitor (20" active TFT, $1600 \times 1200$ at $60 \mathrm{~Hz}, 16 \mathrm{~ms}$ pixel response time). We opted here for an optical method for achieving isoluminance, rather than a method based, for example, on minimum-motion or flicker, to minimize subjective influences on stimulus intensity (e.g. Chaudhuri and Albright, 1990)

Chromatic and low-luminance contrast stimuli were presented in separate blocks of trials. Each block contained 200 stimuli with equal probability of IC and NC conditions. Each participant completed four chromatic contrast blocks (two for pink on green and two for green on pink) and four luminance contrast blocks. Block order was counterbalanced across participants. Stimuli were presented for $500 \mathrm{~ms}$ with an inter-stimulus interval ranging between 800 and $1200 \mathrm{~ms}$. A central fixation dot of the same chromaticity or luminance as the 'pacmen' was displayed on the computer screen throughout the entire block of trials. The participant's task was a two-alternative forced choice that required the discrimination between IC and NC presence on each trial via a right-handed button press. Stimulus delivery and behavioural response collection were controlled by E-prime 2 software (Psychology Software Tools Inc., Pittsburgh, Pennsylvania, USA; www.pstnet.com/eprime). During the experiment, participants took regular breaks between blocks of trials to maintain high concentration and prevent fatigue.

\subsection{EEG acquisition and pre-processing}


Continuous EEG was acquired at $1024 \mathrm{~Hz}$ through a 128 -channel Biosemi ActiveTwo AD-box (http://www.biosemi.com) referenced to the common mode sense (CMS; active electrode) and grounded to the driven right leg (DRL; passive electrode), which functions as a feedback loop driving the average potential across the electrode montage to the amplifier zero (full details, including a diagram of this circuitry, can be found at http://www.biosemi.com/faq/cms\&drl.htm). Prior to epoching, the continuous EEG was filtered (second-order Butterworth with -12db/octave roll-off; $0.1 \mathrm{~Hz}$ high-pass; $60 \mathrm{~Hz}$ low-pass; $50 \mathrm{~Hz}$ notch). The filters were computed linearly in both forward and backward directions to eliminate phase shifts.

EEG epochs were time-locked to the presentation of visual stimuli and spanned 100ms prestimulus and 500ms post-stimulus. Epochs with amplitude deviations in excess of $\pm 60 \mu \mathrm{V}$ at any channel, with the exception of those labelled as 'bad' due to poor electrode-skin contact or damage, were considered artefacts and were excluded. Likewise, trials with blinks or other transients were excluded off-line based on vertical and horizontal electro-oculograms. Data from 'bad' channels were interpolated using 3D splines (Perrin et al., 1987). VEP data were also re-calculated against an average reference and baseline-corrected using the pre-stimulus interval. For each participant, four VEPs were calculated: $I C_{C}, N C_{C}, I C_{L}$, and $N C_{L}$. The mean ( \pm s.e.m.) number of accepted $E E G$ epochs for each of these conditions was $365 \pm 8,365 \pm 7,368 \pm 7$, and $374 \pm 5$, respectively. These values did not significantly differ $\left(F_{(3,9)}=2.1 ; p>0.15\right)$. Differences were then calculated between the IC and $N C$ conditions for each type of contrast, separately, in order to isolate brain activity associated with IC sensitivity (Murray et al., 2002). Hereafter, we refer to these differences for the chromatic contrast and luminance contrast conditions as the $\mathrm{C}_{\mathrm{IC} \text { effect }}$ and $\mathrm{L}_{\mathrm{I} \text { effect, }}$ respectively.

\subsection{VEP analyses}

VEP analyses were performed using the Cartool freeware (http://sites.google.com/site/fbmlab/cartool/cartooldownload; Brunet et al., 2011) as well as STEN utilities (http://unil.ch/line/home/menuinst/about-the-line/software--analysis-tools.html). Effects 
were identified using an analysis framework referred to as electrical neuroimaging (Koenig et al., 2014; Michel and Murray, 2012; Michel et al., 2004; Murray et al., 2008; Tzovara et al., 2012). These electrical neuroimaging analyses allowed us to differentiate between effects following from modulations in the strength of responses of statistically indistinguishable brain generators, alterations in the configuration of the active generators (inferred from the topography of the electric field at the scalp), as well as the latency shifts in brain processes across experimental conditions. We conducted two sets of VEP analyses. The first set of analyses focused on identifying the timing of the IC effect for each type of stimulus contrast. The second set of analyses focused on identifying $\underline{\text { mechanistic differences }}$ between the IC effects for each type of stimulus contrast after first removing any confounding effects of differences in the general VEP response latency (see Results).

To ascertain the timing of the IC effect, we first analyzed the VEP voltage waveform data from all electrodes as a function of time post-stimulus onset in a series of paired comparisons (ttests) between IC and NC responses to the chromatic contrast and luminance contrast conditions, separately. Temporal auto-correlation at individual electrodes was corrected through the application of a 20 contiguous data-point temporal criterion ( $20 \mathrm{~ms}$ at $1024 \mathrm{~Hz}$ sampling) for the persistence of differential effects (Guthrie and Buchwald, 1991). Similarly, spatial correlation was addressed by considering as reliable only those effects that entailed $>10 \%$ of the electrodes from the 128 -channel montage (i.e. $\geq 12$ electrodes). These combined criteria were applied to (partially) correct for multiple comparisons. Our use of an average reference receives support from biophysical laws as well as from the implicit re-centring of VEP data to it when performing source estimations (discussed in Brunet et al., 2011). Analyses of the VEP voltage waveform data (vs. the average reference) are presented here to provide a clearer link between the canonical VEP analysis approaches and the electrical neuroimaging framework (Figure 2). While the former give a visual impression of specific effects within the dataset, our conclusions are principally based on reference-independent global measures of the electric field at the scalp. The measure we used for assessing the timing of the IC effect was the Global Field Power (GFP), which quantifies the electric field strength (Lehmann and Skrandies, 
1980). This measure is equivalent to the standard deviation of the voltage potential values across the entire electrode montage at a given time point and represents a reference-independent measure of the VEP strength (Koenig and Melie-García, 2010; Koenig et al., 2014; Murray et al., 2008). The GFP was used to determine when the $C_{\text {ICeffect }}$ and $\mathrm{L}_{\text {ICeffect }}$ responses were themselves each significantly different from baseline (i.e. when the IC effect itself was reliable). This was achieved using the socalled Topographic Consistency Test (TCT) (Koenig and Melie-García, 2010), which tests the observed GFP against a distribution based on permutations of electrode values in space across the montage (i.e. a pure noise response that preserves the statistical properties of the original dataset). In the case of the IC effect, the TCT provides a statistical determination of when a reliable response begins for each type of stimulus contrast.

The second set of analyses focused on ascertaining whether there are mechanistic differences in the IC effect across stimulus contrasts. As expected from the findings of Foxe et al. (2008; cf. their Figure 3), and confirmed by visual inspection of the VEPs, VEPs were generally shifted $\sim 30 \mathrm{~ms}$ later for luminance vs. chromatic contrast conditions, independently of the presence/absence of ICs (Figure 2). Thus, we re-aligned each participant's VEPs from each condition to the peak of the P1 component (Supplementary Figure 1). The P1 component was defined as the first peak in the GFP that was characterized by a posterior bilateral positivity and fronto-central negativity in the VEP topography (see also Murray et al.(2001)). The re-alignment procedure was semi-automated using a customized Python script (Python Software Foundation; Python Language Reference, version 2.6. Available at http://www.python.org) in the following way: First, the maximal GFP value over the 0$100 \mathrm{~ms}$ period was identified. All values smaller than this maximum were set to zero. Next, the maximal GFP value and its latency over the $105-182$ ms period were identified. To ensure that this GFP maximum was indeed indexing a P1 response, the polarity of nine posterior and nine frontal electrodes at the latency of this GFP peak amplitude was also queried. If at least 7 posterior electrodes measured positive potentials and at least 7 frontal electrodes measured negative potentials, then the GFP maximum was indeed considered as a P1, and the VEP was temporally re- 
aligned (i.e. the peak was labelled as $100 \mathrm{~ms}$ post-stimulus onset) as a consequence. We likewise assessed whether or not differences in peak P1 latency were correlated with reaction times. There was no evidence for reliable correlations (all $p^{\prime} s>0.35$ ), despite reaction times in the chromatic condition being $\sim 30 \mathrm{~ms}$ faster than those in the luminance condition $(488 \pm 13 \mathrm{~ms}$ vs. $522 \pm 15 \mathrm{~ms}$; $\left.\mathrm{t}_{(11)}=5.44 ; p<0.01\right)$.

The re-aligned VEP data from the $I C_{C}$ and $N C_{C}$ conditions, as well as the $I C_{L}$ and $N C_{L}$ conditions were then subtracted separately to obtain the $C_{\mid c e f f e c t}$ and the $L_{1 \text { Ceffect }}$ (Figure 3a). The remainder of the analyses compared these differential responses. First, modulations in the VEP strength (quantified by GFP) were tested using a millisecond-by-millisecond paired t-test in conjunction with the abovementioned 20 contiguous data-point temporal criterion ( $20 \mathrm{~ms}$ at $1024 \mathrm{~Hz}$ sampling) for significant effects to correct for multiple contrasts. While GFP provides an assay of the VEP strength, it is inherently insensitive to spatial (i.e. topographic) differences in the VEPs.

In order to test the VEP topography independently of its strength, we used Global Dissimilarity (DISS)(Lehmann and Skrandies, 1980). DISS is equivalent to the square root of the mean of the squared difference between the potentials measured at each electrode for different conditions, normalized by the instantaneous GFP. It is also directly related to the (spatial) correlation between two normalized vectors (cf. Appendix in Murray et al., 2008). We also performed a nonparametric randomization test (colloquially termed "TANOVA"; Murray et al. (2008)). The DISS value at each time point was compared to an empirical distribution derived from permuting the condition label of the data from each subject. Because changes in topography forcibly follow from changes in the configuration of the underlying active sources (Lehmann et al., 1987), this analysis reveals whether, and if so, when illusory contour sensitivity defined by chromatic vs. luminance contrast activated distinct brain networks. Next, the collective post-stimulus group-average VEPs were subjected to a topographic cluster analysis based on a hierarchical clustering algorithm (Murray et al., 2008). This clustering identifies stable electric field topographies (hereafter template maps). The clustering is sensitive exclusively to topographic modulations, because the data are first normalized 
by their instantaneous GFP. The optimal number of temporally-stable VEP clusters (i.e. the minimal number of maps that accounts for the greatest variance in the data) was determined using a modified Krzanowski-Lai criterion (Murray et al., 2008). The clustering makes no assumption regarding the orthogonality of the derived template maps (De Lucia et al., 2010; Koenig et al., 2014; Pourtois et al., 2008). Template maps identified in the group-average VEP were then submitted to a fitting procedure wherein each time point of each single-subject VEP was labelled according to the template map with which it best correlated spatially (Murray et al., 2008), so as to statistically test the relative presence of each template map in the moment-by-moment scalp topography of the VEPs and the differences in such across conditions. These values can be expressed as the probability of a given template map yielding a higher spatial correlation in the single-subject data from each condition. A statistical analysis of these values was performed using ANOVA, with experimental condition ( $\mathrm{C}_{\mathrm{ICeffect}}$ and $\left.\mathrm{L}_{\mathrm{I} \text { Ceffect }}\right)$ and map as within-subject factors.

Finally, we estimated the intracranial sources of the VEPs as a function of time using a distributed linear inverse solution (ELECTRA) and applying the local autoregressive average (LAURA) regularization approach to address the non-uniqueness of the inverse problem (Grave de Peralta Menendez et al., 2004, 2001; Michel et al., 2004). The inverse solution algorithm is based on biophysical principles derived from the quasi-static Maxwell's equations, most notably the fact that independently of the volume conductor model used to describe the head, only irrotational and not solenoidal currents contribute to the EEG (Grave de Peralta Menendez et al., 2004, 2001). As part of the regularization strategy, homogenous regression coefficients in all directions and within the whole solution space were used. LAURA uses a realistic head model, and the solution space included 3005 nodes, selected from a grid equally distributed within the gray matter of the Montreal Neurological Institute's average brain (grey matter segmentation courtesy of Grave de Peralta Menendez and Gonzalez Andino; http://www.electrical-neuroimaging.ch/). The head model and lead field matrix were generated with the Spherical Model with Anatomical Constraints (SMAC; Spinelli et al. (2000) as implemented in Cartool. As an output, LAURA provides current density measures; their scalar values 
were evaluated at each node. Prior basic and clinical research has documented and discussed in detail the spatial accuracy of this inverse solution (Gonzalez Andino et al., 2005; Grave de Peralta Menendez et al., 2004; Martuzzi et al., 2009). The source estimations were calculated after first averaging across time for each subject and condition. The relevant time interval was determined based on the above topographic clustering analysis used to identify periods of stable VEP topography. These data matrices were then contrasted using a paired t-test. To partially correct for multiple testing, we applied a significance threshold of $p<0.05$ and a spatial-extent criterion $\left(k_{E}\right)$ of >25 contiguous solution points (Bourquin et al., 2013; De Lucia et al., 2010; Knebel and Murray, 2012; Matusz et al., 2015; Toepel et al., 2015).

\section{Results}

\subsection{Behaviour}

Accuracy rates for all conditions were between $92 \%$ and $100 \%$, with a mean of $97 \%$, providing no evidence that IC perception varied with the type of stimulus contrast. These values also provide a clear indication that inducers from all conditions were visible and readily perceived. Behavioural data were analyzed using inverse efficiency scores (Townsend and Ashby 1978, 1983), which are mean reaction times divided by percent correct responses and therefore account for any potential differences due to a speed-accuracy trade-off across conditions. Inverse efficiency was analyzed using a $2 \times 2$ repeated measures analysis of variance (ANOVA) with factors illusory contour presence (IC/NC) and contrast type (C/L). There was a main effect of contour presence, with generally greater efficiency for IC than NC stimuli $\left(508 \pm 13\right.$ vs. $535 \pm 13 \mathrm{~ms} ; F_{(1,11)}=36.49 ; p<0.01$; $\left.\eta_{p}{ }^{2}=0.77\right)$. Paired t-tests confirmed this was the case for both chromatic $\left(t_{(11)}=4.05 ; p<0.0002\right)$ and luminance contrast $\left(t_{(11)}=8.20 ; p<0.0001\right)$ conditions. There was also a main effect of contrast type, with generally greater efficiency for chromatic than luminance contrast $(503 \pm 11$ vs. $540 \pm 15 \mathrm{~ms}$; $\left.F_{(1,11)}=24.83 ; p<0.01 ; \eta_{p}^{2}=0.69\right)$, which was confirmed by paired t-tests $\left(I C_{C} V s . I C_{L}: t_{(11)}=4.38 ; p<0.0012\right.$ and $\mathrm{NC}_{\mathrm{C}}$ vs. $\left.\mathrm{NC}_{\mathrm{L}}: \mathrm{t}_{(11)}=5.32 ; \mathrm{p}<0.0003\right)$. Finally, there was a significant interaction between the factors 
of illusory contour presence and contrast type $\left(F_{(1,11)}=7.12 ; p<0.01 ; \eta_{p}{ }^{2}=0.39\right)$. This interaction was due to a larger IC vs. NC difference for stimuli defined by luminance than chromatic contrast $(31 \pm 4 \mathrm{~ms}$ vs. $\left.22 \pm 6 \mathrm{~ms} ; \mathrm{t}_{(11)}=3.79 ; p<0.005\right)$. Future research will likely need to parametrically vary either the perceived strength of or accuracy in discriminating ICs defined by chromatic versus luminance contrast and their consequences on the corresponding brain mechanisms.

\subsection{Timing of the IC effect as a function of type of stimulus contrast}

We first provide the results of the analysis carried out on the non-subtracted data (as well as prior to the temporal re-alignment), separately for responses to luminance contrast and chromatic contrast conditions, as to provide an indication of the timing of the IC effect for each type of contrast (see Materials and Methods for details). Figure 2a displays VEPs from an exemplar parieto-occipital scalp site (PO7) from luminance contrast and chromatic contrast conditions in response to IC and NC stimuli. Both luminance and chromatic contrast stimuli elicited robust VEPs of similar magnitude, though generally the VEPs were delayed in response to luminance vs. chromatic contrast stimuli. Figure $\mathbf{2 b}$ displays the IC-NC difference waveform (i.e. the IC effect) at the same scalp site. Both types of contrast elicited robust IC effects with what appears to be similar morphologies, and again with what appears to be a general delay for luminance vs. chromatic contrast. Figure $\mathbf{2 c}$ displays the corresponding $p$-value of the IC vs. NC paired contrast as a function of time. Albeit focused on a single electrode, this analysis indicates that the IC effect for stimuli defined by luminance contrast onsets at $83 \mathrm{~ms}$, while for stimuli defined by chromatic contrast it does so at $95 \mathrm{~ms}$. Applying the TCT analysis to the IC effect for each type of stimulus contrast revealed that the responses onset at $85 \mathrm{~ms}$ for stimuli defined by luminance contrast and at $93 \mathrm{~ms}$ for stimuli defined by chromatic contrast (Figure 2d). In general, the timing of the IC effects for both types of stimulus contrast are consistent with prior findings using high-contrast luminance stimuli (Knebel and Murray, 2012; Murray and Herrmann, 2013; Murray et al., 2002).

\subsection{Mechanistic comparison of IC effects across types of stimulus contrast}


Our primary interest was the mechanistic comparison of IC processes for stimuli defined by chromatic versus luminance contrast. We therefore calculated the IC effect for each contrast type, i.e. $C_{1 \text { Ceffect }}$ and $L_{1 \text { Ceffect }}$ and performed all subsequent analyses by statistically comparing these VEP differences. To prevent any general temporal differences in the VEP responses to luminance and chromatic contrast from confounding the identification of mechanistic differences between the $\mathrm{L}_{1 \text { Ceffect }}$ and $\mathrm{C}_{1 \text { Ceffect, }}$ we first temporally aligned the VEPs to the peak of the P100 component. Figure 3a displays the $\mathrm{L}_{\text {ICeffect }}$ and $\mathrm{C}_{\text {ICeffect }}$ at electrode PO7 after alignment to the P100 component. While the morphology of the IC effect for both types of stimulus contrast (as detailed above) were generally similar, differences appeared to onset already after the initial $\sim 100 \mathrm{~ms}$ post-stimulus. We examined this statistically using GFP and DISS (Figure $\mathbf{3 b}$ and $\mathbf{3} \mathbf{c}$, respectively). There was no evidence for reliable differences in response strength (GFP) between the $L_{1 \text { Ceffect }}$ and $C_{\text {ICeffect }}$. There were no periods of time when the t-test result yielded a $p$-value $\leq 0.05$ for at least 11 continuous data points. By contrast, these IC effects significantly differed in their topography (DISS) over the $109-130 \mathrm{~ms}$ poststimulus period. In order to better understand the nature of these topographic differences, the group-averaged $\mathrm{L}_{\mathrm{I} \text { ceffect }}$ and $\mathrm{C}_{\mathrm{ICeffect}}$ data were submitted to a topographic cluster analysis. Two template maps were identified to be present over the $108-159$ ms post-stimulus period, consistent with the above analyses. These template maps were then fitted to the single-subject $L_{1}$ effect and $C_{1 C e f f e c t}$ VEPs, so as to determine the amount of time over the $108-159 m s$ period when each template map better correlated spatially with the single-subject data. These values were then submitted to a $2 \times 2$ ANOVA (condition $\times$ template map), which revealed a significant interaction $\left(F_{(1,11)}=9.97\right.$; $\left.p=0.009 ; \eta_{p}^{2}=0.48\right)$. One map predominated $L_{\text {ICeffect }}$ responses, whereas another map predominated $\mathrm{C}_{\text {ICeffect }}$ responses (Figure $\mathbf{3 d}$ ). These results indicate collectively that distinct brain networks contribute to IC sensitivity during the $108-159$ ms post-stimulus period when stimuli are defined by luminance vs. chromatic contrast.

\subsection{Source Estimations}


Finally, distributed source estimations were performed on the single-subject data after first averaging the $C_{1 \text { Ceffect }}$ and $L_{1 \text { ceffect }}$ VEPs over the $108-159 m s$ period (i.e. the period identified in the above topographic clustering analysis). Figure 4 a displays the mean source estimations for the $\mathrm{C}_{\text {ICeffect }}$ and $L_{1 C e f f e c t}$ (top and bottom, respectively). For the $C_{1 \text { ceffect, }}$ there were prominent sources localised within the bilateral lateral occipital cortex and the calcarine sulcus. For the $L_{1 \text { ceffect }}$, prominent sources were localised within the bilateral calcarine sulcus and the left lateral occipital cortex. For each node within the solution space, a paired t-test was performed. We considered as reliable those clusters wherein each node yielded a significant test $(p<0.05)$ and was moreover located within a cluster of at least 25 significant nodes ( $\mathrm{k}_{\mathrm{E}}>25$ nodes). The results showed significant differences in the generators underlying the $\mathrm{C}_{1 \text { Ceffect }}$ and $\mathrm{L}_{1}$ effect, located principally within right lateral occipital cortices and extending posteriorly to the occipital pole and the calcarine sulcus bilaterally as well as superiorly to inferior parietal cortices bilaterally (Figure 4b). In all the significant voxels, sources were stronger for the $C_{1 \text { ceffect }}$ than the $L_{1 \text { Ceffect }}$. The maximal difference $\left(t_{(11)}=-4.87 ; p<0.0005\right)$ was located within the right lateral occipital cortex $(43,-79,2 \mathrm{~mm}$ according to the Talairach and Tournoux 1988 coordinate system). This pattern of differences in source estimations is consistent with the topographic modulations described above insofar as a subset of commonly active regions was differentially active in the $\mathrm{C}_{\text {ICeffect }}$ than in the $\mathrm{L}_{\mathrm{ICeffect}}$

\section{Discussion}

This study revealed that brain mechanisms underlying IC sensitivity vary according with whether the eliciting stimuli are defined by chromatic versus luminance contrast. Robust perceptions of ICs were achieved with both types of stimulus contrast, but with greater perceptual efficiency when ICs were defined by chromatic than luminance contrast. The VEP data revealed highly similar IC effects in terms of their timing across the two types of stimulus contrast, despite a general latency advantage for responses to the isoluminant chromatic contrast than for the low-luminance achromatic contrast. Critically, the topography, but not magnitude, of the IC effect differed as a 
function of the type of stimulus contrast. Partially distinct circuits were found to contribute to IC sensitivity across contrast types starting as early as $110 \mathrm{~ms}$ post-stimulus. Distributed source estimations further indicated there to be a greater involvement of the LOC as well as V1/V2 to the $\mathrm{C}_{\mathrm{ICeffect}}$ than to the $\mathrm{L}_{\mathrm{I} \text { effect }}$. Collectively, these data provide the first evidence for multiple brain circuits contributing to IC perception.

\subsection{Brain mechanisms of IC sensitivity across types of contrast}

The timing of both the $\mathrm{C}_{\text {ICeffect }}$ as well as the $\mathrm{L}_{\mathrm{ICeffect}}$ is highly consistent with prior findings that used high-contrast achromatic stimuli. For example, Murray et al. (2002) identified the onset of the IC effect at 90ms and localized it to the LOC. This 'brain signature' of IC sensitivity is robust across a range of stimulus/perceptual variations: contrast polarity (Murray et al., 2002; see also Dresp et al., 1996 for psychophysical findings), modal vs. amodal completion (i.e. whether or not there was a perceived brightness enhancement; Murray et al., 2004), accuracy in discriminating convex versus concave shapes created by the ICs (Murray et al., 2006), or the stimulus type eliciting the ICs (Kanizsa-type stimuli or misaligned gratings; Knebel and Murray, 2012;Mendola et al., 1999). The present $\mathrm{C}_{\mathrm{I} \text { Ceffect }}$ and $\mathrm{L}_{\mathrm{C} \text { effect }}$ have yet again corroborated this brain response pattern. Across both conditions, the IC effect onset again at $\sim 90 \mathrm{~ms}$ and originated within the LOC (albeit significantly more strongly for the $C_{1 \text { Ceffect }}$ than for the $\left.L_{1 \text { Ceffect }}\right)$. These results contradict some prior behavioural studies that had suggested that ICS are not perceived (or are at best poorly perceived) under isoluminant chromatic contrast conditions (Li and Guo, 1995; Soriano et al., 1996). However, the task used by Li and Guo (1995) required observers to judge line lengths within a Kanizsa form (i.e. a variant of the Ponzo illusion), rather than the presence/absence of an illusory contour per se. Their paradigm thus did not provide a direct metric of IC sensitivity. By contrast, Ejima and Takahashi (1988) showed that ICs can be reliably perceived when defined by chromatic contrast. Our current results provide direct evidence for the ability of ICS based on both luminance and chromaticity differences to elicit IC perception. 
The timing alone of these IC effects, similar to those reported before (Murray and Herrmann, 2013; Murray et al., 2002), is inconsistent with a purely feedforward nature of the underlying mechanism. As the IC effect follows the VEP onset by several tens of milliseconds, there is ample time for both response propagation across brain regions as well as integration of activity across brain areas containing distinct retinotopic representations (Murray et al., 2001). This temporal lag is particularly important given that the inducers of ICs were spatially distributed across quadrants of the visual field and thus necessitated interhemispheric and intrahemispheric integration and/or convergence of responses in brain regions consisting of neurons with large, bilateral receptive fields. Higher-order visual cortex substrates for IC sensitivity are thus also supported by the progressive increase in neural receptive field size and interhemispheric connectivity across visual cortices from V1 through to the LOC (Smith et al., 2001; see also Box 3 in Murray and Herrmann, 2013). While prior work would indicate that placing all of the inducer elements within one visual hemifield delays the IC effect by approximately 120ms (Brandeis and Lehmann, 1989; Murray et al., 2002), it may be possible to further dissociate circuits for IC sensitivity by capitalising on knowledge concerning photoreceptor distribution across the peripheral retina where it may be more readily possible to target rod-driven IC effects (Ahnelt, 1998).

More generally, the current findings enrich the extant literature in several important ways. First, we demonstrated that the previously reported LOC activity is accompanied by prominent activity within the calcarine sulcus, across both types of stimulus contrast. Second, we provide the first evidence for topographic differences as a function of the type of contrast of the IC-eliciting stimuli. The initial time period of the previously identified IC effect differed in its topography across the $C_{1 \text { Ceffect }}$ and $\mathrm{L}_{\text {ICeffect. }}$ Importantly, we were able to establish that this topographic difference followed from enhanced activity within the right LOC as well as within the bilateral calcarine sulcus (most likely V1/V2; Figure 4) in the case of the $C_{\text {ICeffect. }}$ In these ways, the present study provided evidence that different brain circuits are activated during the initial stages of IC sensitivity according 
to the type of stimulus contrast used (see also Bushnell et al., 2011, for similar evidence in the macaque V4).

\subsection{The role of V1/V2 in IC sensitivity: New insights}

There are several possible accounts for the currently observed activity within V1/V2 that starkly differ between each other in how important a role they attribute to this activity in mediating IC sensitivity. On the one hand, it may be contended that the varieties of contrast used here resulted in greater demands on, and thus stronger activation of, top-down attentional control processes for the purpose of distinguishing between ICs and NCs (e.g. (Ding et al., 2014). Studies in humans that reported V1/V2 activity have similarly used highly demanding tasks (Larsson et al., 1999; Maertens and Pollmann, 2005), making it difficult to establish whether the activity within V1/V2 contributes directly to IC or is instead a result of task demands. In line with the latter, sensitivity within V1 to ICS was found when the IC forms were perceived as moving vertically across the visual field, but substantially less so when the ICs were static (Seghier et al., 2000). Likewise, effects within V1/V2 were observed in studies where the task necessitated discriminating fine features of the IC shape (i.e., task demands were high; e.g., curvature, as in Maertens and Shapley(2008) or crispness, as in Stanley and Rubin (2003). However, the present data speak against the proposition of the role of V1/V2 activation being linked to task demands alone. First, the near-ceiling performance $(>92 \%$ for all conditions) demonstrates that the task was not particularly demanding for the participants. In line with this, several studies in humans have suggested that IC perception occurs pre-attentively (Davis and Driver, 1994; Mattingley et al., 1997; Vuilleumier et al., 2001) and matures by 8 months of age (Csibra et al., 2000; though see Altschuler et al. (2012). Second, we demonstrated stronger V1/V2 responses in what was an ostensibly easier stimulus condition, i.e. when ICs were induced by the isoluminant chromatic contrast. If predominantly task difficulty was mediating these effects, then stronger responses should have been observed for the low-luminance achromatic contrast condition, whose processing was less efficient. 
We propose that under certain conditions the LOC and V1/V2 give rise to IC sensitivity by operating in concert. Notably, this cooperation does not seem to occur with high-contrast achromatic stimuli. Because LOC and V1/V2 sources were both identified over the same time period, there is no evidence to suggest that one region is mediating responsiveness of the other. This notwithstanding, lesion data in humans and monkeys would indeed suggest that perception of (highcontrast achromatic) ICS relies predominantly on the integrity of the LOC (Sáry et al., 2007; Vuilleumier et al., 2001). Further research in brain-lesioned patients is necessary to determine the exact role of $\mathrm{V} 1 / \mathrm{V} 2$ in IC sensitivity when defined by isoluminant chromatic contrast or lowluminance achromatic contrast.

\subsection{Timing of magnocellular- vs. parvocellular-biased VEPS}

Based on the present evidence, one could contend that the isoluminant chromatic and lowluminance achromatic contrast stimuli used here successfully biased brain responses towards the parvocellular and magnocellular visual pathways, respectively. It is therefore noteworthy that there was a $\sim 30 \mathrm{~ms}$ latency advantage for responses to chromatic than luminance contrast (Figure 2). This result could be considered contrary to the evidence from studies using single-unit recordings that suggest a latency advantage for magnocellular- vs. parvocellular-biased stimuli both in the lateral geniculate nucleus as well as the primary visual cortex (Maunsell and Gibson, 1992; Nowak et al., 1995; Schmolesky et al., 1998; Schroeder et al., 1998). However, single-unit activity does not forcibly reflect the net output response of the entire neuronal population. Likewise, computational modelling suggests that the greater convergence of parvocellular than magnocellular fibres onto primary visual cortex could actually result in an overall faster response to parvocellular inputs (Maunsell et al., 1999). In accord, human VEP and fMRI studies have reported precisely such a latency advantage for chromatic vs. luminance stimuli (Foxe et al., 2008; Liu et al., 2006). For example, a clear latency difference is apparent in Figure 3 of Foxe et al. (2008), although their focus was instead on the putative contributions of the two pathways to the C1 component of the VEPs and the potential 
implications thereof for models of attention. While not quantified, the latency shift would appear to be on the order of approximately $30 \mathrm{~ms}$, similarly to our findings. Our study thus provides the first direct evidence for temporally facilitated responses to chromatic and, thus, putatively parvocellularbiased, stimuli. While the neurophysiologic bases for this latency difference as well as for the discrepancies between single-unit and population-based levels of quantification remain to be fully resolved in future research, the present results lay grounds for further investigations of the visual system dynamics and of the 'division of labour' between the two pathways and, thus, for a better understanding of their contribution to visual processing deficits in clinical populations (e.g., Javitt, 2009). That said, it is important to also note that peak latency and amplitude of VEP components, such as P1 and N1, are strongly influenced by low-level stimulus features, including contrast (e.g. Butler et al., 2007), the number and distribution of pixels within an image (Doniger et al., 2002; Foxe et al., 2001), and stimulus location within the visual field (Clark et al., 1995; Murray et al., 2001).

\subsection{Conclusions}

This is the first study that compared the brain mechanisms underlying IC sensitivity to isoluminant chromatic and low-luminance achromatic contrast stimuli. Our results substantially enrich the existing models by revealing that there are partly distinct parallel circuits that can mediate IC sensitivity. It will be important for future research to determine when and how these circuits cooperate or compete during natural vision. 


\section{Acknowledgements}

This work has been supported by the Swiss National Science Foundation (Grant 320030149982 to MMM, Marie-Heim-Vögtlin fellowship PMPDP3_129028 to SCH, as well as the National Centre of Competence in Research project "SYNAPSY: The Synaptic Bases of Mental Disease" [project 51AU40-125759 to MMM]) as well as the Swiss Brain League (2014 Research Prize to MMM). The authors declare no competing financial interests. 


\section{References}

Ahnelt, P.K., 1998. The photoreceptor mosaic. Eye (Lond). 12 ( Pt 3b, 531-40. doi:10.1038/eye.1998.142

Altschuler, T.S., Molholm, S., Russo, N.N., Snyder, A.C., Brandwein, A.B., Blanco, D., Foxe, J.J., 2012. Early electrophysiological indices of illusory contour processing within the lateral occipital complex are virtually impervious to manipulations of illusion strength. Neuroimage $59,4074-$ 85. doi:10.1016/j.neuroimage.2011.10.051

Bourquin, N.M.-P., Murray, M.M., Clarke, S., 2013. Location-independent and location-linked representations of sound objects. Neuroimage 73, 40-9. doi:10.1016/j.neuroimage.2013.01.026

Brandeis, D., Lehmann, D., 1989. Segments of event-related potential map series reveal landscape changes with visual attention and subjective contours. Electroencephalogr. Clin. Neurophysiol. 73, 507-19.

Brunet, D., Murray, M.M., Michel, C.M., 2011. Spatiotemporal analysis of multichannel EEG: CARTOOL. Comput. Intell. Neurosci. 2011, 813870. doi:10.1155/2011/813870

Bushnell, B.N., Harding, P.J., Kosai, Y., Bair, W., Pasupathy, A., 2011. Equiluminance cells in visual cortical area v4. J. Neurosci. Off. J. Soc. Neurosci. 31, 12398-12412. doi:10.1523/JNEUROSCI.1890-11.2011

Butler, P.D., Martinez, A., Foxe, J.J., Kim, D., Zemon, V., Silipo, G., Mahoney, J., Shpaner, M., Jalbrzikowski, M., Javitt, D.C., 2007. Subcortical visual dysfunction in schizophrenia drives secondary cortical impairments. Brain 130, 417-30. doi:10.1093/brain/awl233

Chaudhuri, A., Albright, T.D., 1990. A new technique for estimating chromatic isoluminance in humans and monkeys. Vis. Neurosci. 5, 605-608.

Clark, V.P., Fan, S., Hillyard, S.A., 1995. Identification of Early Visual Evoked Potential Generators by Retinotopic and Topographic Analyses 187.

Csibra, G., Davis, G., Spratling, M.W., Johnson, M.H., 2000. Gamma oscillations and object processing in the infant brain. Science 290, 1582-5.

Davis, G., Driver, J., 1994. Parallel detection of Kanizsa subjective figures in the human visual system. Nature 371, 791-793. doi:10.1038/371791a0

De Lucia, M., Michel, C.M., Murray, M.M., 2010. Comparing ICA-based and single-trial topographic ERP analyses. Brain Topogr. 23, 119-27. doi:10.1007/s10548-010-0145-y

Ding, Y., Martinez, A., Qu, Z., Hillyard, S.A., 2014. Earliest stages of visual cortical processing are not modified by attentional load. Hum. Brain Mapp. 35, 3008-3024.

Doniger, G.M., Foxe, J.J., Murray, M.M., Higgins, B. a, Javitt, D.C., 2002. Impaired visual object recognition and dorsal/ventral stream interaction in schizophrenia. Arch. Gen. Psychiatry 59, 1011-20.

Dresp, B., Salvano-Pardieu, V., Bonnet, C., 1996. Illusory form with inducers of opposite contrast polarity: evidence for multistage integration. Percept. Psychophys. 58, 111-124.

Ejima, Y., Takahashi, S., 1988. Illusory contours induced by isoluminant chromatic patterns. Vision Res. 28, 1367-1377.

Foxe, J.J., Doniger, G.M., Javitt, D.C., 2001. Early visual processing deficits in schizophrenia: impaired P1 generation revealed by high-density electrical mapping. Neuroreport 12, 3815-20.

Foxe, J.J., Strugstad, E.C., Sehatpour, P., Molholm, S., Pasieka, W., Schroeder, C.E., McCourt, M.E., 2008. Parvocellular and magnocellular contributions to the initial generators of the visual evoked potential: high-density electrical mapping of the "C1" component. Brain Topogr. 21, 1121. doi:10.1007/s10548-008-0063-4

Gharat, A., Baker, C.L., 2012. Motion-defined contour processing in the early visual cortex. J. Neurophysiol. 108, 1228-1243. doi:10.1152/jn.00840.2011

Gonzalez Andino, S.L., Michel, C.M., Thut, G., Landis, T., Grave de Peralta, R., 2005. Prediction of response speed by anticipatory high-frequency (gamma band) oscillations in the human brain. Hum. Brain Mapp. 24, 50-8. doi:10.1002/hbm.20056 
Grave de Peralta Menendez, R., Gonzalez Andino, S., Lantz, G., Michel, C.M., Landis, T., 2001. Noninvasive localization of electromagnetic epileptic activity. I. Method descriptions and simulations. Brain Topogr. 14, 131-7.

Grave de Peralta Menendez, R., Murray, M.M., Michel, C.M., Martuzzi, R., Gonzalez Andino, S.L., 2004. Electrical neuroimaging based on biophysical constraints. Neuroimage 21, 527-39. doi:10.1016/j.neuroimage.2003.09.051

Gregory, R.L., 1977. Vision with isoluminant colour contrast: 1.A projection technique and observations. Perception 6, 113-119.

Grosof, D.H., Shapley, R.M., Hawken, M.J., 1993. Macaque V1 neurons can signal "illusory" contours. Nature 365, 550-552. doi:10.1038/365550a0

Guthrie, D., Buchwald, J.S., 1991. Significance testing of difference potentials. Psychophysiology 28, 240-244.

Hochstein, S., Ahissar, M., 2002. View from the top: hierarchies and reverse hierarchies in the visual system. Neuron 36, 791-804.

Ishihara, S., 1972. Tests for color-blindness. Kanehara Shuppan Co., Ltd., Tokyo.

Javitt, D.C., 2009. When doors of perception close: bottom-up models of disrupted cognition in schizophrenia. Annu. Rev. Clin. Psychol. 5, 249-75. doi:10.1146/annurev.clinpsy.032408.153502

Kanizsa, G., 1976. Subjective contours. Sci. Am. 234, 48-52.

Knebel, J.-F., Javitt, D.C., Murray, M.M., 2011. Impaired early visual response modulations to spatial information in chronic schizophrenia. Psychiatry Res. 193, 168-76. doi:10.1016/j.pscychresns.2011.02.006

Knebel, J.-F., Murray, M.M., 2012. Towards a resolution of conflicting models of illusory contour processing in humans. Neuroimage 59, 2808-17. doi:10.1016/j.neuroimage.2011.09.031

Knebel, J.-F., Toepel, U., Hudry, J., le Coutre, J., Murray, M.M., 2008. Generating controlled image sets in cognitive neuroscience research. Brain Topogr. 20, 284-9. doi:10.1007/s10548-0080046-5

Koenig, T., Melie-García, L., 2010. A method to determine the presence of averaged event-related fields using randomization tests. Brain Topogr. 23, 233-42. doi:10.1007/s10548-010-0142-1

Koenig, T., Stein, M., Grieder, M., Kottlow, M., 2014. A tutorial on data-driven methods for statistically assessing ERP topographies. Brain Topogr. 27, 72-83. doi:10.1007/s10548-0130310-1

Larsson, J., Amunts, K., Gulyás, B., Malikovic, a, Zilles, K., Roland, P.E., 1999. Neuronal correlates of real and illusory contour perception: functional anatomy with PET. Eur. J. Neurosci. 11, 402436.

Lee, T.S., Nguyen, M., 2001. Dynamics of subjective contour formation in the early visual cortex. Proc. Natl. Acad. Sci. U. S. A. 98, $1907-1911$.

Lehmann, D., Ozaki, H., Pal, I., 1987. EEG alpha map series: brain micro-states by space-oriented adaptive segmentation. Electroencephalogr. Clin. Neurophysiol. 67, 271-288.

Lehmann, D., Skrandies, W., 1980. Reference-free identification of components of checkerboardevoked multichannel potential fields. Electroencephalogr. Clin. Neurophysiol. 48, 609-621.

Li, C.Y., Guo, K., 1995. Measurements of geometric illusions, illusory contours and stereo-depth at luminance and colour contrast. Vision Res. 35, 1713-20.

Liu, C.-S.J., Bryan, R.N., Miki, A., Woo, J.H., Liu, G.T., Elliott, M.A., 2006. Magnocellular and parvocellular visual pathways have different blood oxygen level-dependent signal time courses in human primary visual cortex. AJNR. Am. J. Neuroradiol. 27, 1628-1634.

Maertens, M., Pollmann, S., 2005. fMRI reveals a common neural substrate of illusory and real contours in V1 after perceptual learning. J. Cogn. Neurosci. 17, 1553-64. doi:10.1162/089892905774597209

Maertens, M., Shapley, R., 2008. Local determinants of contour interpolation. J. Vis. 8, 3.1-11. doi:10.1167/8.7.3

Martuzzi, R., Murray, M.M., Meuli, R. a, Thiran, J.-P., Maeder, P.P., Michel, C.M., Grave de Peralta Menendez, R., Gonzalez Andino, S.L., 2009. Methods for determining frequency- and region- 
dependent relationships between estimated LFPs and BOLD responses in humans. J. Neurophysiol. 101, 491-502. doi:10.1152/jn.90335.2008

Masuda, T., Matsubara, K., Utsumi, K., Wada, Y., 2015. Material perception of a kinetic illusory object with amplitude and frequency changes in oscillated inducer motion. Vision Res., Perception of Material Properties (Part I) 109, Part , 201-208. doi:10.1016/j.visres.2014.11.019

Mattingley, J.B., Davis, G., Driver, J., 1997. Preattentive filling-in of visual surfaces in parietal extinction. Science 275, 671-4.

Matusz, P.J., Thelen, A., Amrein, S., Geiser, E., Anken, J., Murray, M.M., 2015. The role of auditory cortices in the retrieval of single-trial auditory-visual object memories. Eur. J. Neurosci. doi:10.1111/ejn.12804

Maunsell, J.H., Ghose, G.M., Assad, J.A., McAdams, C.J., Boudreau, C.E., Noerager, B.D., 1999. Visual response latencies of magnocellular and parvocellular LGN neurons in macaque monkeys. Vis. Neurosci. 16, 1-14.

Maunsell, J.H., Gibson, J.R., 1992. Visual response latencies in striate cortex of the macaque monkey. J. Neurophysiol. 68, 1332-1344.

Mendola, J.D., Dale, a M., Fischl, B., Liu, a K., Tootell, R.B., 1999. The representation of illusory and real contours in human cortical visual areas revealed by functional magnetic resonance imaging. J. Neurosci. 19, 8560-72.

Michel, C.M., Murray, M.M., 2012. Towards the utilization of EEG as a brain imaging tool. Neuroimage 61, 371-85. doi:10.1016/j.neuroimage.2011.12.039

Michel, C.M., Murray, M.M., Lantz, G., Gonzalez, S., Spinelli, L., Grave de Peralta, R., 2004. EEG source imaging. Clin. Neurophysiol. Off. J. Int. Fed. Clin. Neurophysiol. 115, 2195-2222. doi:10.1016/j.clinph.2004.06.001

Murray, M.M., Brunet, D., Michel, C.M., 2008. Topographic ERP analyses: a step-by-step tutorial review. Brain Topogr. 20, 249-64. doi:10.1007/s10548-008-0054-5

Murray, M.M., Foxe, D.M., Javitt, D.C., Foxe, J.J., 2004. Setting boundaries: brain dynamics of modal and amodal illusory shape completion in humans. J. Neurosci. 24, 6898-903. doi:10.1523/JNEUROSCI.1996-04.2004

Murray, M.M., Foxe, J.J., Higgins, B. a, Javitt, D.C., Schroeder, C.E., 2001. Visuo-spatial neural response interactions in early cortical processing during a simple reaction time task: a highdensity electrical mapping study. Neuropsychologia 39, 828-44.

Murray, M.M., Herrmann, C.S., 2013. Illusory contours: a window onto the neurophysiology of constructing perception. Trends Cogn. Sci. 17, 471-481. doi:10.1016/j.tics.2013.07.004

Murray, M.M., Imber, M.L., Javitt, D.C., Foxe, J.J., 2006. Boundary completion is automatic and dissociable from shape discrimination. J. Neurosci. 26, 12043-54. doi:10.1523/JNEUROSCI.322506.2006

Murray, M.M., Wylie, G.R., Higgins, B. a, Javitt, D.C., Schroeder, C.E., Foxe, J.J., 2002. The spatiotemporal dynamics of illusory contour processing: combined high-density electrical mapping, source analysis, and functional magnetic resonance imaging. J. Neurosci. 22, 5055-73.

Nieder, a, Wagner, H., 1999. Perception and neuronal coding of subjective contours in the owl. Nat. Neurosci. 2, 660-3. doi:10.1038/10217

Nowak, L.G., Munk, M.H., Girard, P., Bullier, J., 1995. Visual latencies in areas V1 and V2 of the macaque monkey. Vis. Neurosci. 12, 371-384.

Pourtois, G., Delplanque, S., Michel, C., Vuilleumier, P., 2008. Beyond conventional event-related brain potential (ERP): exploring the time-course of visual emotion processing using topographic and principal component analyses. Brain Topogr. 20, 265-277. doi:10.1007/s10548-008-0053-6

Redies, C., Crook, J.M., Creutzfeldt, O.D., 1986. Neuronal responses to borders with and without luminance gradients in cat visual cortex and dorsal lateral geniculate nucleus. Exp. Brain Res. 61, 469-81.

Sáry, G., Chadaide, Z., Tompa, T., Köteles, K., Kovács, G., Benedek, G., 2007. Illusory shape representation in the monkey inferior temporal cortex. Eur. J. Neurosci. 25, 2558-64. doi:10.1111/j.1460-9568.2007.05494.x 
Sáry, G., Köteles, K., Kaposvári, P., Lenti, L., Csifcsák, G., Frankó, E., Benedek, G., Tompa, T., 2008. The representation of Kanizsa illusory contours in the monkey inferior temporal cortex. Eur. J. Neurosci. 28, 2137-46. doi:10.1111/j.1460-9568.2008.06499.x

Schmolesky, M.T., Wang, Y., Hanes, D.P., Thompson, K.G., Leutgeb, S., Schall, J.D., Leventhal, a G., 1998. Signal timing across the macaque visual system. J. Neurophysiol. 79, 3272-8.

Schroeder, C.E., Mehta, a D., Givre, S.J., 1998. A spatiotemporal profile of visual system activation revealed by current source density analysis in the awake macaque. Cereb. Cortex 8, 575-92.

Seghier, M., Dojat, M., Delon-Martin, C., Rubin, C., Warnking, J., Segebarth, C., Bullier, J., 2000. Moving illusory contours activate primary visual cortex: an fMRI study. Cereb. Cortex 10, 66370.

Smith, A.T., Singh, K.D., Williams, A.L., Greenlee, M.W., 2001. Estimating receptive field size from fMRI data in human striate and extrastriate visual cortex. Cereb. Cortex 11, 1182-90.

Song, Y., Baker, C.L., 2007. Neuronal response to texture- and contrast-defined boundaries in early visual cortex. Vis. Neurosci. 24, 65-77. doi:10.1017/S0952523807070113

Soriano, M., Spillmann, L., Bach, M., 1996. The abutting grating illusion. Vision Res. 36, 109-16.

Spinelli, L., Andino, S.G., Lantz, G., Seeck, M., Michel, C.M., 2000. Electromagnetic inverse solutions in anatomically constrained spherical head models. Brain Topogr. 13, 115-25.

Stanley, D. a, Rubin, N., 2003. fMRI activation in response to illusory contours and salient regions in the human lateral occipital complex. Neuron 37, 323-31.

Takahashi, S., Kaihara, T., Takemoto, A., Ido, K., Ejima, Y., 1992. Spectral sensitivities for illusory contour perception: a manifold linkage of chromatic and achromatic cues with the generation of contours. Vision Res. 32, 1709-1718.

Toepel, U., Bielser, M.-L., Forde, C., Martin, N., Voirin, A., le Coutre, J., Murray, M.M., Hudry, J., 2015. Brain dynamics of meal size selection in humans. Neuroimage 113, 133-142. doi:10.1016/j.neuroimage.2015.03.041

Tzovara, A., Murray, M.M., Michel, C.M., De Lucia, M., 2012. A tutorial review of electrical neuroimaging from group-average to single-trial event-related potentials. Dev. Neuropsychol. 37, 518-44. doi:10.1080/87565641.2011.636851

Ungerleider, L.G., Mishkin, M., 1982. Two cortical visual systems, in: Visual Behavior. D.J. Ingle, M.A. Goodale \& R.J.W. Mansfield, pp. 549-586.

von der Heydt, R., Peterhans, E., Baumgartner, G., 1984. Illusory contours and cortical neuron responses. Science 224, 1260-1262.

Vuilleumier, P., Valenza, N., Landis, T., 2001. Explicit and implicit perception of illusory contours in unilateral spatial neglect: behavioural and anatomical correlates of preattentive grouping mechanisms. Neuropsychologia 39, 597-610.

Yoshino, A., Kawamoto, M., Yoshida, T., Kobayashi, N., Shigemura, J., Takahashi, Y., Nomura, S., 2006. Activation time course of responses to illusory contours and salient region: a high-density electrical mapping comparison. Brain Res. 1071, 137-44. doi:10.1016/j.brainres.2005.11.089 


\section{Figure Captions}

Figure 1. Illustration of stimulus conditions. a. Isoluminant chromatic contrast stimuli either appeared pink on green or vice versa. b. Low-luminance achromatic contrast stimuli used pacmen inducers that were slightly darker than the background. Note that the contrast in the figure was modified for ease of visibility. The illusory contour (IC) condition involved inducers whose mouths all faced inward (top row), while the no contour (NC) condition involved inducers whose mouths all faced outward (bottom row). The central fixation point has been enlarged for illustration purposes.

Figure 2. Comparative timing of IC effects. a. Group-averaged ( $N=12)$ VEP waveforms from a left parieto-occipital scalp location (PO7) for all 4 stimulus conditions of the study are displayed. Comparable and robust responses were obtained for all conditions, although shifted later in time for the low-luminance achromatic conditions. b. The group-averaged VEP difference waveforms (IC effect) are shown for the isoluminant chromatic contrast and low-luminance achromatic contrast conditions (red and black traces, respectively, with shading indicating the s.e.m.). c. Paired t-tests were performed between IC and NC responses as a function of time for isoluminant chromatic and low-luminance achromatic contrast, separately (red and black area plots, respectively). Significant IC effects were observed starting at $\sim 90-95 m s$ post-stimulus onset $(p<0.05$ for a minimum of 20 consecutive data points). d. The topographic consistency test was performed using the difference waveforms across the entire electrode montage for isoluminant chromatic and low-luminance achromatic contrast, separately (red and black area plots, respectively). This was done to empirically test for onset of the IC effect using a global and reference-independent VEP measure. Significant IC effects were observed starting at $\sim 90-95$ ms post-stimulus onset (same criterion as above).

Figure 3. Comparative mechanisms of IC effects. a. The group-averaged ( $N=12)$ VEP difference waveforms (IC effect) are shown for the isoluminant chromatic contrast and low-luminance achromatic contrast conditions after first re-aligning the un-subtracted VEPs (see Figure 2a) to the 
peak of the P100 component(red and black traces, respectively, with shading indicating the s.e.m.). b. The group-averaged global field power (GFP) waveforms of the $\mathrm{C}_{\mathrm{ICeffect}}$ and $\mathrm{L}_{\mathrm{I} \text { ceffect }}$ are shown (red and black traces, respectively, with shading indicating the s.e.m.). No statistically reliable differences were observed. c. The global dissimilarity (blue trace) is shown as a function of time. Significant topographic differences between $C_{1 \text { Ceffect }}$ and $L_{1 \text { Ceffect }}$ were observed over the 109-130ms period (pale green area). $\mathbf{d}$. The topographic cluster analysis identified two maps over the $108-159 \mathrm{~ms}$ period. The bar graph shows that one map better account for the $C_{\text {Iceffect }}$ and another for the $\mathrm{L}_{\text {ICeffect }}$, which was quantified as the amount of time over the $108-159$ ms period when each map best correlated spatially with the single-subject data (meanıs.e.m. shown).

Figure 4. Comparative distributed source estimations for IC effects. a. Group-averaged distributed source estimations over the $108-159 \mathrm{~ms}$ period are shown for the $\mathrm{C}_{\text {ICeffect }}$ and $\mathrm{L}_{\mathrm{ICeffect}}$ (top and bottom, respectively). b. Statistical contrast of these source estimations are displayed and revealed reliable differences within the right lateral occipital cortices as well as calcarine sulcus bilaterally. 
a. Isoluminant chromatic

IC

\begin{tabular}{|c|c|c|c|}
\hline$r$ & 9 & ? & $?$ \\
\hline 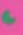 & 2 & $c$ & 3 \\
\hline 3 & $c$ & 0 & 6 \\
\hline ? & C & $?$ & e \\
\hline
\end{tabular}

b. Low-luminance achromatic

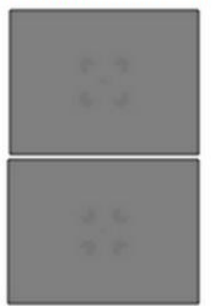


a. Exemplar VEPs

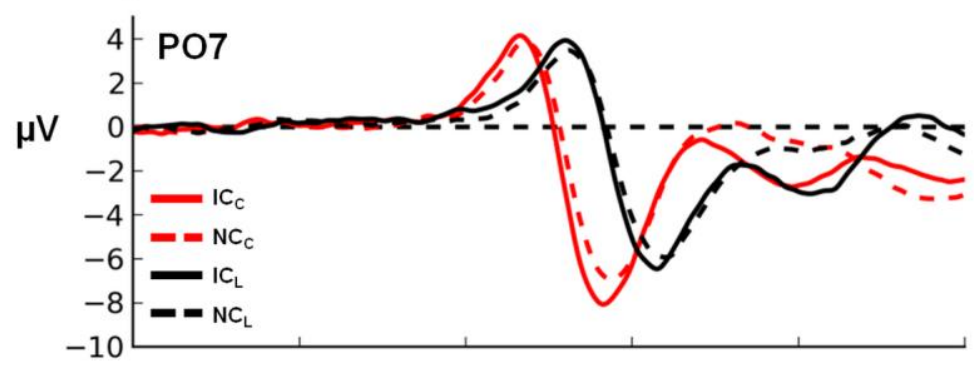

b. VEP difference waveforms

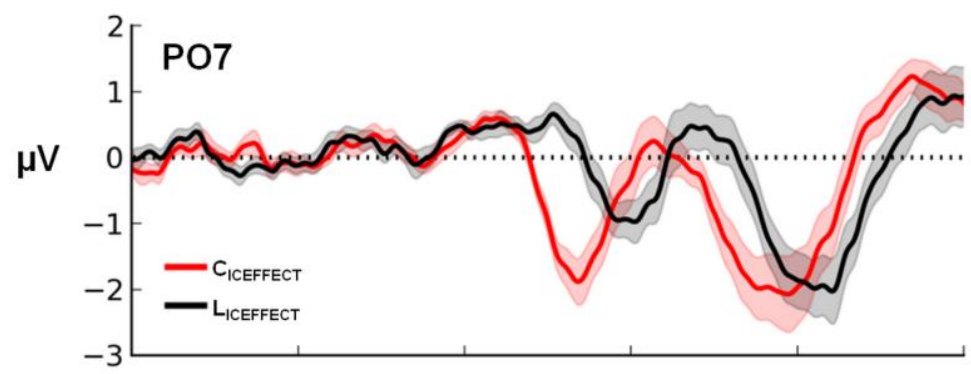

c. T-test (at PO7)

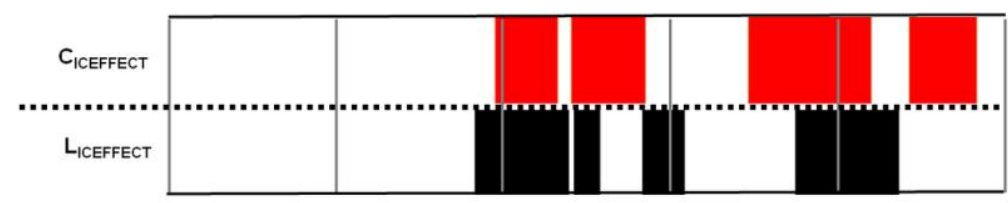

d. Topographic Consistency Test

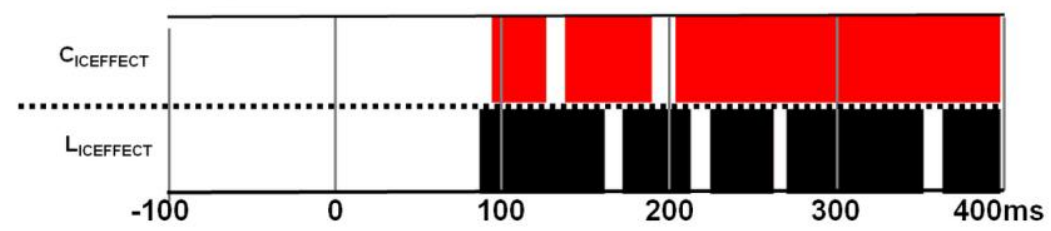


a. VEP difference (post-alignment)

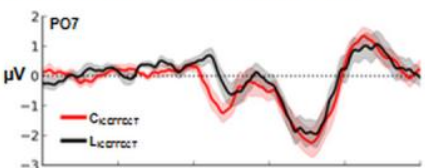

b. Global Field Power

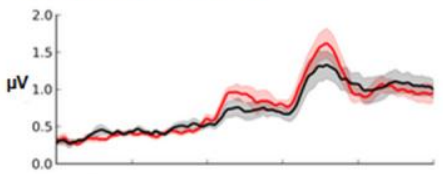

c. TANOVA

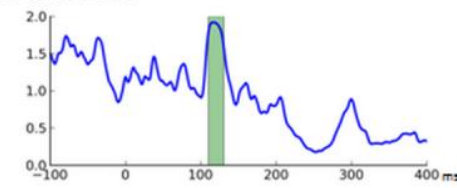

d. Topographic cluster analysis and single-subject fitting (108-159ms)

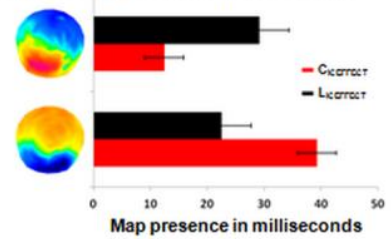


a. Mean source estimations (IC effect 108-159ms)

$c_{\text {eremer }}$
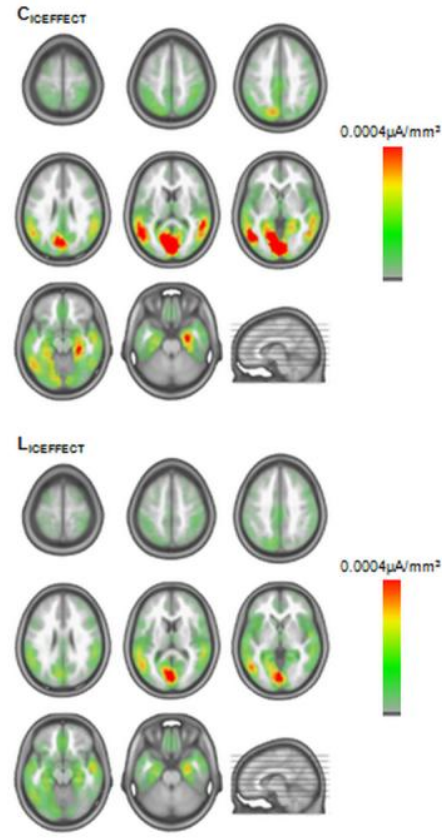

b. Statistical difference

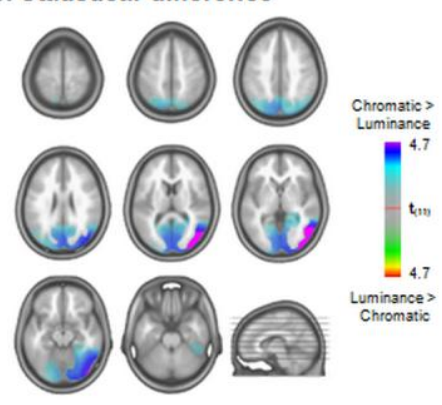

